\title{
A survival model with Birnbaum-Saunders frailty for uncensored and censored cancer data
}

\author{
Jeremias Leão $^{\mathrm{a}, \mathrm{b}}$, Víctor Leiva ${ }^{\mathrm{c}}$, Helton Saulo ${ }^{\mathrm{d}, \mathrm{e}}$ and Vera Tomazella ${ }^{\mathrm{a}}$ \\ ${ }^{a}$ Universidade Federal do Amazonas \\ ${ }^{\mathrm{b}}$ Universidade Federal de Sao Carlos \\ ${ }^{\mathrm{c} S c h o o l ~ o f ~ I n d u s t r i a l ~ E n g i n e e r i n g, ~ P o n t i f i c i a ~ U n i v e r s i d a d ~ C a t o ́ l i c a ~ d e ~ V a l p a r a i ́ s o ~}$ \\ ${ }^{\mathrm{d}}$ Universidade Federal de Goiás \\ ${ }^{\mathrm{e}}$ Universidade de Brasília
}

\begin{abstract}
Survival models with frailty are used when additional data are non-available to explain the occurrence time of an event of interest. This non-availability may be considered as a random effect related to unobserved explanatory variables, or that cannot be measured, often attributed to environmental or genetic factors. We propose a survival model with frailty based on the Birnbaum-Saunders distribution. This distribution has been widely applied to lifetime data. The random effect is the frailty, which is assumed to follow the Birnbaum-Saunders distribution and introduced on the baseline hazard rate to control the unobservable heterogeneity of the patients. We use the maximum likelihood method to estimate the model parameters and evaluate its performance under different censoring proportions by a Monte Carlo simulation study. Two types of residuals are considered to assess the adequacy of the proposed model. Examples with uncensored and censored real-world data sets illustrate the potential applications of the proposed model.
\end{abstract}

\section{Introduction and preliminary notions}

Frailty models are characterized by the inclusion of a random effect containing data that have not been observed or cannot be measured, usually associated with environmental or genetic factors. Also, it may be attributed to data that were not considered in the planning of the study; see Hougaard (1995). The random effect is the frailty, which is introduced in the baseline hazard rate (HR) additively or multiplicatively. The frailty is considered to control the unobservable heterogeneity of the units under analysis. In survival models, the units can be patients with different frailties, that is, those patients who are most frail (or prone) tend to have a disease earlier than the less frail patients. The concept of frailty was introduced by Vaupel, Manton and Stallard (1979) in survival models based on the gamma (GA) distribution.

A number of authors have studied the multiplicative frailty models, which represent a generalization of the Cox regression model; see Cox (1972). Hougaard

Key words and phrases. Birnbaum-Saunders distribution, frailty models, likelihood methods, medical data, Monte Carlo simulation, R software.

Received March 2016; accepted March 2017. 
(1995) and Sinha and Dey (1997) studied multiplicative frailty models from the classical and Bayesian perspectives, respectively. For some specific applications on univariate frailty models, interested readers are refereed to Aalen and Tretli (1999), Henderson and Oman (1999), and Baker and Henderson (2005), whereas the book by Wienke (2011) provides details about frailty models.

Consider Cox (1972)'s model and an unobserved source of heterogeneity, which is not captured by any explanatory variable of this model. An extension of the Cox model can be considered by allowing the HR of a patient to depend on an unobservable random variable $U$, acting multiplicatively on the baseline HR. Consequently, the conditional HR of the time to the event of interest $T$ given $U=u_{i}$, for the patient $i$, at time $t$, can be written as

$$
h_{T \mid U=u_{i}}\left(t ; \boldsymbol{\theta}_{1}, \boldsymbol{\theta}_{2}\right)=u_{i} h_{0}\left(t ; \boldsymbol{\theta}_{1}\right), \quad i=1, \ldots, n, t>0,
$$

where $u_{i}$ is the frailty of the patient $i$ and $h_{0}$ is a baseline HR, with $\boldsymbol{\theta}_{1}$ and $\boldsymbol{\theta}_{2}$ being the parameters of the lifetime and frailty distributions, respectively. Note that Equation (1.1) is known as Clayton (1991)'s model, from which it is possible to observe that the risk of the patient $i$ increases if $u_{i}>1$ and decreases if $u_{i}<1$. The conditional survival function (SF) can be obtained from Equation (1.1) as

$$
S_{T \mid U=u_{i}}\left(t ; \boldsymbol{\theta}_{1}, \boldsymbol{\theta}_{2}\right)=\exp \left(-u_{i} H_{0}\left(t ; \boldsymbol{\theta}_{1}\right)\right), \quad i=1, \ldots, n, t>0,
$$

with $H_{0}\left(t ; \boldsymbol{\theta}_{1}\right)=\int_{0}^{t} h_{0}\left(s ; \boldsymbol{\theta}_{1}\right) \mathrm{d} s$ being the baseline cumulative hazard rate (CHR). Suppose that the time to the event of interest is not completely observed and it can be subject to right censoring. Let $c_{i}$ denote the censoring time and $y_{i}$ the observed time to the event of interest. Then, $t_{i}=\min \left\{y_{i}, c_{i}\right\}$, whereas $\kappa_{i}=I\left(t_{i} \leq c_{i}\right)$ is a censoring indicator such that $\kappa_{i}=1$ if $y_{i}$ is the time to the event of interest and $\kappa_{i}=0$ if it is right censored for the patient $i$. From Equations (1.1) and (1.2), the corresponding log-likelihood function for the vector of model parameters $\boldsymbol{\theta}=$ $\left(\boldsymbol{\theta}_{1}, \boldsymbol{\theta}_{2}\right)^{\top}$ is given by

$$
\ell(\boldsymbol{\theta} ; \boldsymbol{t}, \boldsymbol{\kappa}, \boldsymbol{u})=\sum_{i=1}^{n} \kappa_{i} \log \left(u_{i} h_{0}\left(t_{i}\right)\right)-\sum_{i=1}^{n} u_{i} H_{0}\left(t_{i}\right),
$$

where $\boldsymbol{t}=\left(t_{1}, \ldots, t_{n}\right)^{\top}$ are the observed times to the event of interest, $\boldsymbol{\kappa}=$ $\left(\kappa_{1}, \ldots, \kappa_{n}\right)^{\top}$ is the vector of their censoring indicators, and $\boldsymbol{u}=\left(u_{1}, \ldots, u_{n}\right)^{\top}$ are the frailties of the patients. The log-likelihood function given in Equation (1.3), conditional on the unobserved frailties $\boldsymbol{u}$, establishes the parameter estimation procedure. Note that the frailties must be integrated out (depending on the frailty distribution) to obtain a log-likelihood function for $\boldsymbol{\theta}$ (independent on unobserved quantities) as

$$
\ell(\boldsymbol{\theta} ; \boldsymbol{t}, \boldsymbol{\kappa})=\sum_{i=1}^{n} \kappa_{i} \log \left(h_{T}\left(t_{i} ; \boldsymbol{\theta}\right)\right)+\sum_{i=1}^{n} \log \left(S_{T}\left(t_{i} ; \boldsymbol{\theta}\right)\right),
$$


where $h_{T}$ and $S_{T}$ are the unconditional HR and SF, respectively, which are defined next.

By integrating $S_{T \mid U=u_{i}}(t)$ given in Equation (1.2) on the frailty $U$, we obtain the unconditional (population) SF of $T$, which can be viewed as the (unconditional) SF of patients randomly drawn from the population under study; see Klein and Moeschberger (2003), Aalen, Borgan and Gjessing (2008) and Wienke (2011). Unconditional HR and SF may be obtained with the Laplace transform; see Hougaard (1984). Therefore, in the process of finding distributions for the frailty random variable $U$, natural candidates are distributions possessing an explicit Laplace transform, because it facilitates the use of traditional maximum likelihood (ML) methods for parameter estimation. To obtain the unconditional SF, we integrate out the frailty component as

$$
S_{T}(t ; \boldsymbol{\theta})=\int_{0}^{\infty} S_{T \mid U=u}\left(t ; \boldsymbol{\theta}_{1}\right) f_{U}\left(u ; \boldsymbol{\theta}_{2}\right) \mathrm{d} u,
$$

where $f_{U}$ is the frailty probability density function (PDF) and $S_{T \mid U=u}(t ; \boldsymbol{\theta})$ is the conditional SF given in Equation (1.2). Let $f=f_{U}$ be the frailty PDF and $s=H_{0}\left(t ; \boldsymbol{\theta}_{1}\right)$. Then, we obtain the Laplace transform of the unconditional SF as

$$
S_{T}(t ; \boldsymbol{\theta})=\int_{0}^{\infty} \exp \left(-u H_{0}\left(t ; \boldsymbol{\theta}_{1}\right)\right) f_{U}\left(u ; \boldsymbol{\theta}_{2}\right) \mathrm{d} u=Q\left(H_{0}\left(t ; \boldsymbol{\theta}_{1}\right)\right) .
$$

Note that Equation (1.5) has a similar form as the unconditional SF defined in Equation (1.4). The frailty random variables $U_{i}$ are often considered as independent and with identical distribution.

As noted, the frailty component of the model is random. Then, a distribution can be assumed for the frailty. The GA distribution is often used in applications of frailty models published to date. This is mainly due to the mathematical treatment, because by using the Laplace transform, we obtain closed-form expressions for its unconditional SF and HR. Other natural candidates to the frailty distribution are the inverse Gaussian (IG), lognormal (LN) and Weibull (WE) distributions; see Hougaard (1995). The Birnbaum-Saunders (BS) distribution is right-skewed (asymmetrical), continuous and unimodal. It is also known as the fatigue life distribution and has received considerable attention due to its theoretical arguments, its attractive properties and its relation with the normal distribution; see Birnbaum and Saunders (1969), Leiva and Saunders (2015) and Leiva (2016). The BS distribution has been extensively applied for modeling failure times in engineering, but novel applications in environmental and financial sciences have been also considered; see Kotz, Leiva and Sanhueza (2010), Lemonte (2013), Saulo et al. (2013, 2019), Leiva et al. (2014a, 2014b, 2015a, 2016a, 2016b, 2017), Sánchez et al. (2015), Wanke and Leiva (2015), Garcia-Papani et al. (2017) and Lillo et al. (2018). In addition, the BS distribution has been applied to biological and medical studies; see Barros, Paula and Leiva (2008), Leiva et al. (2015b) and Leao et al. (2017). All of these applications have been conducted by an international, 
transdisciplinary group of researchers. Therefore, such as the GA, IG, LN and WE distributions, another natural candidate to model frailty is the BS distribution.

The objective of this paper is to propose a survival model with frailty BS distributed, which can be an alternative to the GA frailty model and useful to describe censored data, as well as, of course, uncensored data. We consider a reparameterized version of the BS (RBS) distribution proposed by Santos-Neto et al. (2012, 2014, 2016). An argument given to consider this reparameterization is related to the present work. We employ the Laplace transform to find the RBS unconditional SF on the individual frailty. We use the ML method to estimate the model parameters and evaluate its performance with Monte Carlo (MC) simulations. We illustrate the potential applications of the proposed model by means of uncensored and censored medical data related to cancer.

After this introduction and background of frailty models and unconditional HR and SF, Section 2 introduces the RBS frailty model, derives the ML estimators of the model parameters and proposes two residuals. Section 3 studies the performance of the corresponding ML estimators through MC simulations and provides two illustrative examples. Section 4 discusses some conclusions.

\section{Birnbaum-Saunders frailty model}

In this section, we present some properties of the RBS distribution, discuss aspects of model identifiability, introduce the RBS frailty model, estimate its parameters and consider two types of residuals.

The RBS distribution is indexed by the parameters $\mu=\beta\left(1+\alpha^{2} / 2\right)$ and $\delta=2 / \alpha^{2}$, where $\alpha>0$ and $\beta>0$ are the original BS parameters (see Birnbaum and Saunders (1969)), $\mu>0$ is a scale parameter and the mean of the distribution, whereas $\delta>0$ is a shape and precision parameter. The notation $U \sim \operatorname{RBS}(\mu, \delta)$ is used when the random variable $U$ follows such a distribution. The RBS distribution permits us to have an alternative GA frailty model (see Vaupel, Manton and Stallard, 1979) as follows. The mean and variance of $U \sim \operatorname{RBS}(\mu, \delta)$ are given by $\mathrm{E}[U]=\mu$ and $\operatorname{Var}[U]=\mu^{2} / \phi$, respectively, where $\phi=(\delta+1)^{2} /(2 \delta+5)$. Therefore, as mentioned, $\delta$ can be interpreted as a precision parameter, because, for fixed values of $\mu$, when $\delta$ goes to infinity, the variance of $U$ tends to zero. Moreover, for fixed $\mu$, if $\delta$ approaches zero, then $\operatorname{Var}[U]$ tends to $5 \mu^{2}$. Note that $\operatorname{Var}[U]=\mu^{2} / \phi$ is similar to the variance function of the GA distribution, which has a quadratic relation with its mean. Therefore, a frailty model based on the RBS distribution can be a good alternative to the GA frailty model. Besides this, the following additional reasons can be listed to stress the use of the RBS distribution: (i) its mean-based parameterization allows the possibility to analyze data in their original scale, since problems of interpretation may arise when a logarithmic transformation of the data is employed; see Leiva et al. (2014a) and Santos-Neto et al. (2014, 
2016); (ii) it appears to be very competitive for fitting frailty models; (iii) a motivation based on its genesis permits its usage for medical data; see Desmond (1985); (iv) it has an explicit Laplace transform, whose characteristic is not shared by the LN frailty model, although both models belong to the class of log-symmetric distributions, which arises when a random variable has the same distribution as its reciprocal; see Vanegas and Paula (2016a, 2016b); and (v) it allows us to model bimodal data; see details of the logarithmic version of the RBS (log-RBS) model below and in Rieck and Nedelman (1991). If $U \sim \operatorname{RBS}(\mu, \delta)$, then its PDF is given by

$$
\begin{aligned}
f_{U}(u ; \mu, \delta)= & \frac{\exp (\delta / 2) \sqrt{\delta+1}}{4 u^{\frac{3}{2}} \sqrt{\pi \mu}}\left(u+\frac{\delta \mu}{\delta+1}\right) \\
& \times \exp \left(-\frac{\delta}{4}\left(\frac{u(\delta+1)}{\delta \mu}+\frac{\delta \mu}{u(\delta+1)}\right)\right), \quad u>0 .
\end{aligned}
$$

It is possible to show that $k U \sim \operatorname{RBS}(k \mu, \delta)$, with $k>0$, and $1 / U \sim \operatorname{RBS}\left(\mu^{*}, \delta\right)$, where $\mu^{*}=(\delta+1) /(\delta \mu)$. From Equation (2.1), the RBS SF and HR are respectively given by

$$
S_{U}(u ; \mu, \delta)=\frac{1}{2} \Phi\left(\frac{u+\delta(u-\mu)}{2 \sqrt{u(1+\delta) \mu}}\right), \quad u>0,
$$

and

$$
h_{U}(u ; \mu, \delta)=\frac{\exp \left(-\frac{(-\delta \mu+\delta u+u)^{2}}{4(\delta+1) \mu u}\right)(\delta \mu+\delta u+u)}{(\pi \mu(\delta+1))^{\frac{1}{2}} 2 \mu^{\frac{1}{2}} u^{\frac{3}{2}} \Phi\left(\frac{u+\delta(u-\mu)}{2 \sqrt{u(1+\delta) \mu}}\right)}, \quad u>0,
$$

where $\Phi$ is the cumulative distribution function (CDF) of the standard normal distribution or $\mathrm{N}(0,1)$ distribution. Note that if $U=\exp (V) \sim \operatorname{RBS}(\mu, \delta)$, then $V \sim \log -\operatorname{RBS}(\sqrt{2 / \delta}, \log (\delta \mu /(\delta+1)))$, that is, the $\log -\mathrm{RBS}$ distribution is obtained. The mean of $V$ is $\mathrm{E}[V]=\log (\delta \mu /(\delta+1))$. Based on the the log-RBS moment generating function, its asymptotic variance (which has no closed form) is, as $\delta$ goes to $\infty, \operatorname{Var}[V]=2 / \delta-1 / \delta^{2}$, whereas that, in contrast, as $\delta$ approaches zero, $\operatorname{Var}[V]=4\left(\log ^{2}(2 / \sqrt{\delta})+2-2 \log (2 / \sqrt{\delta})\right)$. The distribution of $V$ is symmetric around $\mu$, unimodal for $\delta \geq 0.5$ and bimodal for $\delta<0.5$; see Rieck and Nedelman (1991) and Leiva (2016).

Figure 1 displays some shapes for the PDF, SF and HR of $U \sim \operatorname{RBS}(1, \delta)$, and some shapes for the PDF of $V=\log (U)$. Note that a unimodal behavior is detected for the PDF of $U$ and different degrees of asymmetry and kurtosis, whereas the HR of $U$ has increasing and decreasing shapes, such as the GA distribution, but also an inverse bathtub shape. Moreover, the bimodality property is noticed in the PDF of $V$.

An important aspect in frailty models to be studied is their identifiability. In the case of proportional hazard models with frailty, it is necessary that the random 

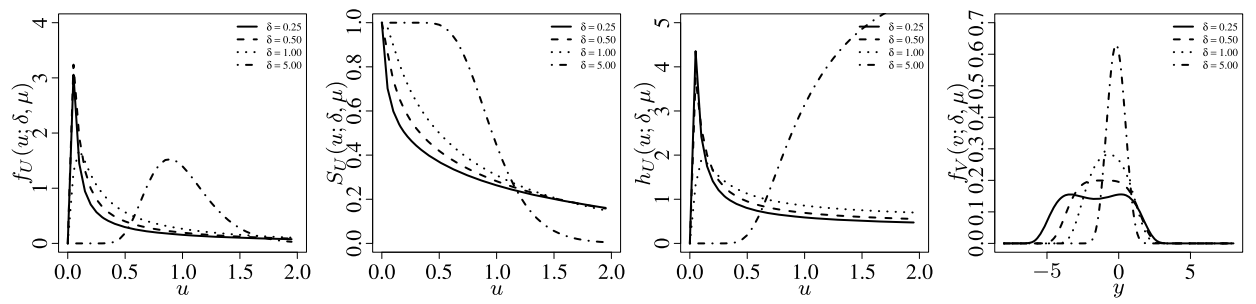

Figure 1 Plots of PDF (left), SF (left-center) and HR (right-center) of the RBS distribution, and $P D F($ right) of the log-RBS distribution for $\mu=1$, with the indicated value of $\delta$.

effect distribution has a finite mean for the model to be identifiable; see Elbers and Ridder (1982). Thus, it is convenient to have a distribution mean fixed at a finite value in order to keep the identifiability of the frailty model, which for simplicity, we assume equal to one. Therefore, we consider that the frailty $U$ has the RBS distribution with parameters $\mu=1$ and $\delta$, where $\mathrm{E}[U]=1$ and $\operatorname{Var}[U]=(2 \delta+$ $5) /(\delta+1)^{2}$. Note that the variance quantifies the amount of heterogeneity among patients.

The Laplace transform for the RBS distribution with parameters $\mu=1$ and $\delta$ is given by

$$
Q(s)=\frac{\exp \left(\frac{\delta}{2}(1-\sqrt{\delta+4 s+1} / \sqrt{\delta+1})\right)(\sqrt{\delta+4 s+1}+\sqrt{\delta+1})}{2 \sqrt{\delta+4 s+1}} .
$$

From Equation (1.5) and evaluating Equation (2.2) at $s=H_{0}(t)$, we obtain the unconditional SF under the RBS frailty as

$$
\begin{aligned}
S_{T}(t) & =\frac{\exp \left(\frac{\delta}{2}\left(1-\sqrt{\delta+4 H_{0}(t)+1} / \sqrt{\delta+1}\right)\right)\left(\sqrt{\delta+4 H_{0}(t)+1}+\sqrt{\delta+1}\right)}{2 \sqrt{\delta+4 H_{0}(t)+1}}, \\
t & >0 .
\end{aligned}
$$

Then, from Equation (2.3), the corresponding unconditional HR is obtained by

$$
\begin{aligned}
h_{T}(t) & =h_{0}(t)\left(\frac{\delta\left(\delta+\sqrt{\delta+1} \sqrt{\delta+4 H_{0}(t)+1}+4 H_{0}(t)+3\right)+2}{\left(\delta+4 H_{0}(t)+1\right)\left(\delta+\sqrt{\delta+1} \sqrt{\delta+4 H_{0}(t)+1}+1\right)}\right), \\
t & >0 .
\end{aligned}
$$

Here, $h_{0}(t)$ is assumed to be specified up to a few unknown parameters, which are related to a distribution assumed for the baseline HR. For example, one can assume an exponential (EXP), LN or WE distribution. However, a parametric distribution assumption is not always desirable, because such an assumption may be difficult to verify. Note that the EXP distribution has been extensively used to model the 
baseline HR due to its simplicity or when the HR must be constant for each patient; see Lawless (2003). Therefore, we use the EXP distribution as baseline hazard, which has $h_{0}(t)=\lambda$ and $H_{0}(t)=\lambda t$, for $t>0$. Thus, from Equation (2.4), the unconditional HR under RBS frailty reduces to

$$
h_{T}(t)=\frac{\lambda(\delta(\delta+\sqrt{\delta+1} \sqrt{\delta+4 \lambda t+1}+4 \lambda t+3)+2)}{(\delta+4 \lambda t+1)(\delta+\sqrt{\delta+1} \sqrt{\delta+4 \lambda t+1}+1)}, \quad t>0,
$$

where $\lambda$ is the average HR of each patient. From Equation (2.5), the unconditional SF under RBS frailty is given by

$$
\begin{aligned}
S_{T}(t) & =\frac{\exp \left(\frac{1}{2} \delta(1-\sqrt{\delta+4 \lambda t+1} / \sqrt{\delta+1})\right)(\sqrt{\delta+1}+\sqrt{\delta+4 \lambda t+1})}{2 \sqrt{\delta+4 \lambda t+1}}, \\
t & >0
\end{aligned}
$$

Note that Equations (2.3) and (2.4) can be easily applied to different baselines other than the EXP one. Indeed, we also assume a WE baseline in Section 3.

Consider $n$ patients providing pairs of times and right censoring indicators $\left(t_{1}, \kappa_{1}\right), \ldots,\left(t_{n}, \kappa_{n}\right)$, with $t_{i}$ and $\kappa_{i}$ being the elements of the vectors $\boldsymbol{t}$ and $\boldsymbol{\kappa}$ defined in Equation (1.3), respectively. Moreover, consider the RBS frailty model given by Equations (2.5) and (2.6) with parameter vector $\boldsymbol{\theta}=(\delta, \lambda)^{\top}$. Therefore, the corresponding log-likelihood function under uninformative censoring can be expressed as

$$
\begin{aligned}
\ell(\boldsymbol{\theta} ; \boldsymbol{t}, \boldsymbol{\kappa}) & \\
= & \frac{n \delta}{2}-\frac{\delta}{2 \sqrt{\delta+1}} \sum_{i=1}^{n} \sqrt{\delta+4 \lambda t_{i}+1}-\sum_{i=1}^{n} \kappa_{i} \log \left(\delta+4 \lambda t_{i}+1\right) \\
& -\sum_{i=1}^{n} \log \left(2 \sqrt{\delta+4 \lambda t_{i}+1}\right)+\sum_{i=1}^{n} \log \left(\sqrt{\delta+1}+\sqrt{\delta+4 \lambda t_{i}+1}\right) \\
& +\sum_{i=1}^{n} \kappa_{i} \log \left(\lambda\left(\delta\left(\delta+\sqrt{(\delta+1)\left(\delta+4 \lambda t_{i}+1\right)}+4 \lambda t_{i}+3\right)+2\right)\right) \\
& -\sum_{i=1}^{n} \kappa_{i} \log \left(\delta+\sqrt{(\delta+1)\left(\delta+4 \lambda t_{i}+1\right)}+1\right) .
\end{aligned}
$$

Then, the first derivatives of the log-likelihood function given in Equation (2.7) with respect to the two parameters are obtained to establish the score vector $\dot{\ell}(\boldsymbol{\theta})=$ $\partial \ell(\boldsymbol{\theta}) / \partial \boldsymbol{\theta}$. For the sake of simplicity, we define $\tau_{i, 1}=\delta+4 \lambda t_{i}+1$ and $\tau_{i, 2}=$ $\delta+\sqrt{(\delta+1) \tau_{i, 1}}+4 \lambda t_{i}+3$. Then, elements of the score vector are expressed 
as

$$
\begin{aligned}
\frac{\partial \ell(\boldsymbol{\theta})}{\partial \delta}= & \frac{n}{2}-\sum_{i=1}^{n} \frac{\kappa_{i}+1}{\tau_{i, 1}}-\sum_{i=1}^{n} \frac{\kappa_{i}\left(\frac{2 \delta+4 \lambda t_{i}+2}{2 \sqrt{(\delta+1) \tau_{i, 1}}}+1\right)}{\delta+\sqrt{(\delta+1) \tau_{i, 1}}+1} \\
& +\sum_{i=1}^{n} \frac{\kappa_{i}\left(\delta+\delta\left(\frac{2 \delta+4 \lambda t_{i}+2}{2 \sqrt{(\delta+1) \tau_{i, 1}}}+1\right)+\sqrt{(\delta+1) \tau_{i, 1}}+4 \lambda t_{i}+3\right)}{\delta \tau_{i, 2}+2} \\
& -\left(\frac{\delta}{4 \sqrt{\delta+1}}\right) \sum_{i=1}^{n} \frac{1}{\sqrt{\tau_{i, 1}}}+\left(\frac{\delta}{4(\delta+1)^{3 / 2}}-\frac{1}{2 \sqrt{\delta+1}}\right) \sum_{i=1}^{n} \sqrt{\tau_{i, 1}} \\
\frac{\partial \ell(\boldsymbol{\theta})}{\partial \lambda=} & -\sum_{i=1}^{n} \frac{4\left(\kappa_{i}+1\right) t_{i}}{\tau_{i, 1}}-\sum_{i=1}^{n} \frac{2(\delta+1) \kappa_{i} t_{i}}{\sqrt{(\delta+1) \tau_{i, 1}}\left(\delta+\sqrt{(\delta+1) \tau_{i, 1}}+1\right)} \\
& +\sum_{i=1}^{n} \frac{\kappa_{i}\left(\delta \tau_{i, 2}+\delta \lambda\left(\frac{2(\delta+1) t_{i}}{\left.\left.\sqrt{(\delta+1) \tau_{i, 1}}+4 t_{i}\right)+2\right)}\right.\right.}{\lambda\left(\delta \tau_{i, 2}+2\right)} \\
& -\left(\frac{\delta}{4 \sqrt{\delta+1}}\right) \sum_{i=1}^{n} \frac{t_{i}}{\sqrt{\tau_{i, 1}}}+\sum_{i=1}^{n} \frac{2 t_{i}}{\sqrt{\tau_{i, 1}}\left(\sqrt{\delta+1}+\sqrt{\tau_{i, 1}}\right)} .
\end{aligned}
$$

ML equations generated from $\dot{\boldsymbol{\ell}}(\boldsymbol{\theta})=\mathbf{0}_{2 \times 1}$, for estimating $\delta$ and $\lambda$, must be solved with an iterative method for non-linear optimization problems, where $\mathbf{0}_{2 \times 1}$ is a $2 \times 1$ vector of zeros. Specifically, the ML estimates of the RBS frailty model parameters can be obtained by using a quasi-Newton non-linear optimization algorithm with numeric derivatives known as the Broyden-Fletcher-Goldfarb-Shanno (BFGS); see Nocedal and Wright (1999) and Lange (2001). The BFGS method is implemented in the R software by the functions optim and optimx; see www.Rproject.org and (2016).

In this case, standard regularity conditions (see Cox and Hinkley (1974)) are fulfilled, if the parameters are within the parameter space. Hence, the estimators $\widehat{\delta}$ and $\widehat{\lambda}$ are consistent and bivariate normal distributed, asymptotically, with means $\delta$ and $\lambda$, respectively, and asymptotic covariance matrix of $\widehat{\boldsymbol{\theta}} \boldsymbol{\Sigma}_{\widehat{\boldsymbol{\theta}}}$ say, which may be computed from the expected Fisher information matrix associated with the RBS frailty model, $\mathcal{I}(\boldsymbol{\theta})$ say. Then, recalling $\boldsymbol{\theta}=(\delta, \lambda)^{\top}$,

$$
\sqrt{n}(\widehat{\boldsymbol{\theta}}-\boldsymbol{\theta}) \stackrel{\mathcal{D}}{\rightarrow} \mathrm{N}_{2}\left(\mathbf{0}_{2 \times 1}, \boldsymbol{\Sigma}_{\widehat{\boldsymbol{\theta}}}=\mathcal{J}(\boldsymbol{\theta})^{-1}\right), \quad \text { as } n \rightarrow \infty,
$$

where $\stackrel{\mathcal{D}}{\rightarrow}$ means convergence in distribution and

$$
\mathcal{J}(\boldsymbol{\theta})=\lim _{n \rightarrow \infty} \frac{1}{n} \mathcal{I}(\boldsymbol{\theta}) .
$$

Here, $\widehat{\mathcal{I}}(\boldsymbol{\theta})^{-1}$ is a consistent estimator of $\boldsymbol{\Sigma}_{\widehat{\theta}}$. The expected Fisher information matrix may be approximated by its observed Fisher information matrix; see Efron 
and Hinkley (1978). The diagonal elements of the inverse of this matrix can be used to approximate the associated standard errors. The observed information matrix for the RBS frailty model is given as follows.

Let $T_{1}, \ldots, T_{n}$ be a random sample from the RBS frailty model and $t_{1}, \ldots, t_{n}$ their observations. From the log-likelihood function given in Equation (2.7), we have that the observed information matrix of the RBS frailty model is given by

$$
\boldsymbol{I}(\boldsymbol{\theta})=\left(\begin{array}{ll}
I_{\delta \delta} & I_{\delta \lambda} \\
I_{\delta \lambda} & I_{\lambda \lambda}
\end{array}\right)
$$

where $I_{\theta_{i} \theta_{j}}=-\partial^{2} \ell(\boldsymbol{\theta}) / \partial \theta_{i} \theta_{j}$, for $i, j=1,2$, with $\theta_{1}=\delta$ and $\theta_{2}=\lambda$. Therefore,

$$
\begin{aligned}
& I_{\delta \delta}=\sum_{i=1}^{n} \frac{\left(\kappa_{i}+1\right)}{\tau_{i, 1}^{2}} \\
& -\sum_{i=1}^{n} \kappa_{i}\left(\frac{\frac{1}{\sqrt{(\delta+1) \tau_{i, 1}}}-\frac{\left(2 \delta+4 \lambda t_{i}+2\right)^{2}}{4\left((\delta+1) \tau_{i, 1}\right)^{3 / 2}}}{\delta+\sqrt{(\delta+1) \tau_{i, 1}}+1}-\frac{\left(\frac{2 \delta+4 \lambda t_{i}+2}{2 \sqrt{(\delta+1) \tau_{i, 1}}}+1\right)^{2}}{\left(\delta+\sqrt{(\delta+1) \tau_{i, 1}}+1\right)^{2}}\right) \\
& +\sum_{i=1}^{n} \kappa_{i}\left(\frac{\frac{2 \delta+4 \lambda t_{i}+2}{\sqrt{(\delta+1) \tau_{i, 1}}}+\delta\left(\frac{1}{\sqrt{(\delta+1) \tau_{i, 1}}}-\frac{\left(2 \delta+4 \lambda t_{i}+2\right)^{2}}{4\left((\delta+1) \tau_{i, 1}\right)^{3 / 2}}\right)+2}{\delta \tau_{i, 2}+2}\right. \\
& \left.-\frac{\left(\delta+\delta\left(\frac{2 \delta+4 \lambda t_{i}+2}{2 \sqrt{(\delta+1) \tau_{i, 1}}}+1\right)+\sqrt{(\delta+1) \tau_{i, 1}}+4 \lambda t_{i}+3\right)^{2}}{\left(\delta \tau_{i, 2}+2\right)^{2}}\right) \\
& -\frac{1}{2} \delta\left(\frac{\sum_{i=1}^{n} \frac{1}{2 \sqrt{\tau_{i, 1}}}}{(\delta+1)^{3 / 2}}-\frac{3 \sum_{i=1}^{n} \sqrt{\tau_{i, 1}}}{4(\delta+1)^{5 / 2}}+\frac{\sum_{i=1}^{n}+\frac{1}{4 \tau_{i, 1} 3 / 2}}{\sqrt{\delta+1}}\right) \\
& +\frac{\sum_{i=1}^{n} \sqrt{\tau_{i, 1}}}{2(\delta+1)^{3 / 2}}-\sum_{i=1}^{n}\left(\frac{\frac{1}{4(\delta+1)^{3 / 2}}+\frac{1}{4 \tau_{i, 1}^{3 / 2}}}{\sqrt{\delta+1}+\sqrt{\tau_{i, 1}}}+\frac{\left(\frac{1}{2 \sqrt{\delta+1}}+\frac{1}{2 \sqrt{\tau_{i, 1}}}\right)^{2}}{\left(\sqrt{\delta+1}+\sqrt{\tau_{i, 1}}\right)^{2}}\right) \\
& -\frac{\sum_{i=1}^{n} \frac{1}{2 \sqrt{\tau_{i, 1}}}}{\sqrt{\delta+1}} \\
& I_{\delta \lambda}=\sum_{i=1}^{n} \frac{4\left(\kappa_{i}+1\right) t_{i}}{\tau_{i, 1}^{2}} \\
& -\sum_{i=1}^{n}\left(\frac{\kappa_{i}\left(\frac{2 t_{i}}{\sqrt{(\delta+1) \tau_{i, 1}}}-\frac{(\delta+1) t_{i}\left(2 \delta+4 \lambda t_{i}+2\right)}{\left((\delta+1) \tau_{i, 1}\right)^{3 / 2}}\right)}{\delta+\sqrt{(\delta+1) \tau_{i, 1}}+1}\right.
\end{aligned}
$$




$$
\begin{aligned}
& \left.-\frac{2(\delta+1) \kappa_{i} t_{i}\left(\frac{2 \delta+4 \lambda t_{i}+2}{2 \sqrt{(\delta+1) \tau_{i, 1}}}+1\right)}{\sqrt{(\delta+1) \tau_{i, 1}}\left(\delta+\sqrt{(\delta+1) \tau_{i, 1}}+1\right)^{2}}\right) \\
& +\sum_{i=1}^{n}\left(\frac{\kappa_{i}\left(\frac{2(\delta+1) t_{i}}{\sqrt{(\delta+1) \tau_{i, 1}}}+\delta\left(\frac{2 t_{i}}{\sqrt{(\delta+1) \tau_{i, 1}}}-\frac{(\delta+1) t_{i}\left(2 \delta+4 \lambda t_{i}+2\right)}{\left((\delta+1) \tau_{i, 1}\right)^{3 / 2}}\right)+4 t_{i}\right)}{\delta\left(\delta+\sqrt{(\delta+1) \tau_{i, 1}}+4 \lambda t_{i}+3\right)+2}\right. \\
& -\left(\delta \kappa _ { i } ( \frac { 2 ( \delta + 1 ) t _ { i } } { \sqrt { ( \delta + 1 ) \tau _ { i , 1 } } } + 4 t _ { i } ) \left(\delta+\delta\left(\frac{2 \delta+4 \lambda t_{i}+2}{2 \sqrt{(\delta+1) \tau_{i, 1}}}+1\right)\right.\right. \\
& \left.\left.\left.+\sqrt{(\delta+1) \tau_{i, 1}}+4 \lambda t_{i}+3\right)\right) /\left(\delta \tau_{i, 2}+2\right)^{2}\right) \\
& +\left(\frac{\delta}{4(\delta+1)^{3 / 2}}-\frac{1}{2 \sqrt{\delta+1}}\right) \sum_{i=1}^{n} \frac{2 t_{i}}{\sqrt{\tau_{i, 1}}}+\left(\frac{\delta}{2 \sqrt{\delta+1}}\right) \sum_{i=1}^{n} \frac{t_{i}}{\tau_{i, 1}^{3 / 2}} \\
& -\sum_{i=1}^{n}\left(\frac{t_{i}}{\tau_{i, 1}^{3 / 2}\left(\sqrt{\delta+1}+\sqrt{\tau_{i, 1}}\right)}+\frac{2 t_{i}\left(\frac{1}{2 \sqrt{\delta+1}}+\frac{1}{2 \sqrt{\tau_{i, 1}}}\right)}{\sqrt{\tau_{i, 1}}\left(\sqrt{\delta+1}+\sqrt{\tau_{i, 1}}\right)^{2}}\right), \\
& I_{\lambda \lambda}=-\sum_{i=1}^{n}\left(\frac{\kappa_{i}\left(\delta \tau_{i, 2}+\delta \lambda\left(\frac{2(\delta+1) t_{i}}{\sqrt{(\delta+1) \tau_{i, 1}}}+4 t_{i}\right)+2\right)}{\lambda^{2}\left(\delta \tau_{i, 2}+2\right)}\right. \\
& +\frac{\kappa_{i}\left(2 \delta\left(\frac{2(\delta+1) t_{i}}{\sqrt{(\delta+1) \tau_{i, 1}}}+4 t_{i}\right)-\frac{4 \delta(\delta+1)^{2} \lambda t_{i}^{2}}{\left((\delta+1) \tau_{i, 1}\right)^{3 / 2}}\right)}{\lambda\left(\delta \tau_{i, 2}+2\right)} \\
& \left.-\frac{\delta \kappa_{i}\left(\frac{2(\delta+1) t_{i}}{\sqrt{(\delta+1) \tau_{i, 1}}}+4 t_{i}\right)\left(\delta \tau_{i, 2}+\delta \lambda\left(\frac{2(\delta+1) t_{i}}{\sqrt{(\delta+1) \tau_{i, 1}}}+4 t_{i}\right)+2\right)}{\lambda\left(\delta \tau_{i, 2}+2\right)^{2}}\right) \\
& +\sum_{i=1}^{n} \frac{16\left(\kappa_{i}+1\right) t_{i}^{2}}{\tau_{i, 1}^{2}}+\sum_{i=1}^{n}\left(\frac{4(\delta+1)^{2} \kappa_{i} t_{i}^{2}}{\left((\delta+1) \tau_{i, 1}\right)^{3 / 2}\left(\delta+\sqrt{(\delta+1) \tau_{i, 1}}+1\right)}\right. \\
& \left.-\frac{4(\delta+1) \kappa_{i} t_{i}^{2}}{\tau_{i, 1}\left(\delta+\sqrt{(\delta+1) \tau_{i, 1}}+1\right)^{2}}\right)+\left(\frac{\delta}{2 \sqrt{\delta+1}}\right) \sum_{i=1}^{n} \frac{4 t_{i}^{2}}{\tau_{i, 1}^{3 / 2}} \\
& +\sum_{i=1}^{n}\left(\frac{4 t_{i}^{2}}{\tau_{i, 1}^{3 / 2}\left(\sqrt{\delta+1}+\sqrt{\tau_{i, 1}}\right)} \frac{4 t_{i}^{2}}{\tau_{i, 1}\left(\sqrt{\delta+1}+\sqrt{\tau_{i, 1}}\right)^{2}}\right) .
\end{aligned}
$$


Thus, from Equation (2.8), we have that an approximate $100 \times(1-\varrho) \%$ confidence region for $\boldsymbol{\theta}$ is given by

$$
\mathcal{R}=\left\{\boldsymbol{\theta} \in \mathbb{R}^{2}:|\widehat{\boldsymbol{\theta}}-\boldsymbol{\theta}|^{\top} \widehat{\boldsymbol{\Sigma}}_{\boldsymbol{\theta}}^{-1}|\widehat{\boldsymbol{\theta}}-\boldsymbol{\theta}| \leq \chi_{1-\varrho}^{2}(2)\right\}, \quad 0<\varrho<1,
$$

where $\chi_{1-\varrho}^{2}(2)$ denotes the $100 \times(1-\varrho)$ th quantile of the chi-squared distribution with two degrees of freedom and $\widehat{\boldsymbol{\Sigma}}_{\theta}$ is an estimate of $\boldsymbol{\Sigma}_{\theta}$. Confidence bands for the RBS frailty model parameters can be obtained by means of the region provided in Equation (2.9).

Goodness of fit of the RBS frailty model may be assessed by residual analysis. First, we propose to use the generalized Cox-Snell (GCS) residual (see Cox and Snell, 1968) given by

$$
r_{i}^{\mathrm{GCS}}=-\log \left(\widehat{S}_{T}\left(t_{i}\right)\right), \quad i=1, \ldots, n,
$$

where $\widehat{S}_{T}$ is the corresponding estimated SF. The GCS residual defined in Equation (2.10) has an $\operatorname{EXP}(1)$ distribution when the frailty model is correctly specified, regardless of the frailty model considered. Second, we propose to use the quantile (QS) residual (see Smith, 1985 and Dunn and Smyth, 1996), which is often employed in generalized additive models for location, scale and shape; see Stasinopoulos and Rigby (2007). The QS residual is given by

$$
r_{i}^{\mathrm{QS}}=\Phi^{-1}\left(\widehat{S}_{T}\left(t_{i}\right)\right), \quad i=1, \ldots, n,
$$

where $\Phi^{-1}$ is the inverse function of the $\mathrm{N}(0,1) \mathrm{CDF}$ and $\widehat{S}_{T}$ is as in Equation (2.10). The QS residual defined in Equation (2.11) has a $\mathrm{N}(0,1)$ distribution if the frailty model is correctly specified for any frailty model considered.

\section{Numerical applications}

In this section, we carry out a simulation study to evaluate the performance of the ML estimators of the RBS frailty model parameters with EXP baseline HR. Then, we illustrate the proposed methodology by applying it to two real-world medical data sets. The first (uncensored) data set refers to a leukemia cancer study introduced by Feigl and Zelen (1965), whereas the second (censored) data set comes from a lung cancer trial presented in Kalbfleisch and Prentice (2002).

Given the frailty component, the times to the event of interest are independent and follow a proportional hazard model. We compare the proposed RBS frailty model (under two different baseline distributions: EXP and WE, which are selected because of their simplicity in modeling lifetimes) and the GA frailty model (under the same baseline distributions). We assess the impact of the frailty model on its variance. Then, we find the model that provides the best fit to the data. To make sure that the model is identifiable, we consider $U \sim \mathrm{GA}(1 / \gamma, 1 / \gamma)$ in the GA frailty model; see Wienke (2011). 


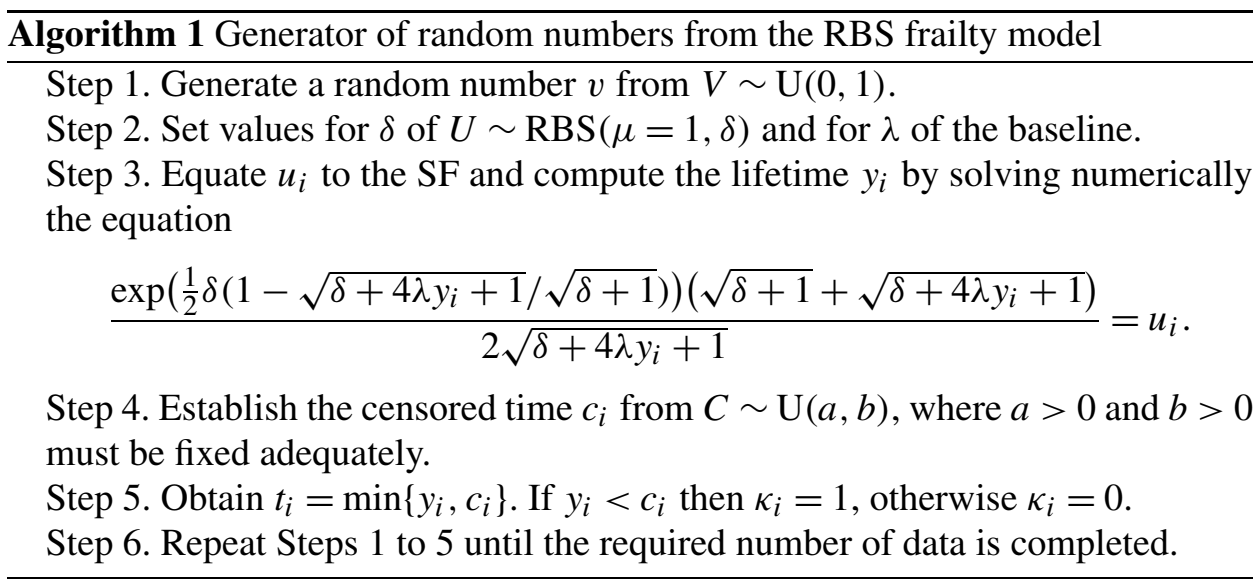

\section{Simulation study}

The scenario considers the following: sample size $n \in\{30,150,400,600\}$, censoring proportion $p \in\{0.00,0.10,0.25,0.40\}$, values for the parameters $\delta \in$ $\{0.25,0.50,1.50,2.50\}$ — which is related to heterogeneity—and $\lambda \in\{1.0,3.0$, $10.0\}$, under $5000 \mathrm{MC}$ replications. We consider the EXP distribution as baseline, which, as it is well known, has the same HR for all $\lambda>0$. Note that, based on the probability integral transform, the RBS frailty CDF follows a $\mathrm{U}(0,1)$ distribution. Then, the RBS frailty SF is $\mathrm{U}(0,1)$ distributed as well. Random number generation from the RBS frailty model is performed following Algorithm 1. In step \#2 of this algorithm, we use the function uniroot of the R software to get the root of the equation; see Brent (1973). For each value of the parameter, sample size and censoring proportion, we report the empirical values for the bias and mean squared error (MSE) of the ML estimators in Tables 1, 2 and 3. From theses tables, note that, as the sample size increases, the ML estimators become more efficient, as expected. We can also note that, as the censoring proportion increases, the performance of the estimator of $\delta$-the shape parameter-deteriorates, which means that the presence of censoring introduces a bias in the ML estimators, as also expected. In addition, we observe that, as $\delta$ increases, the bias of the ML estimator increases as well. However, the performance of the estimator of $\lambda$-the scale parameter of the baseline distribution-improves. Furthermore, we observe that, as the value of $\lambda$ increases, the performance of its estimator decreases. In short, efficiency of the ML estimators is ratified by our simulation study. Therefore, in general, all of these results show the good performance of the ML estimators of the corresponding parameters.

\section{First case study: Leukemia cancer data}

The data set corresponds to the survival times of 33 patients who died from acute myelogenous leukemia, whose set we name "leukemia data". The counting of 
Table 1 Empirical bias (with MSEs in parentheses) of the ML estimators of $\delta$ and $\lambda=1.0$ from the $R B S$ frailty model under the indicated $n, \delta$ and $p$ for simulated data

\begin{tabular}{|c|c|c|c|c|c|}
\hline \multirow[b]{2}{*}{$n$} & \multirow[b]{2}{*}{$\delta$} & \multicolumn{2}{|c|}{$p=0.00$} & \multicolumn{2}{|c|}{$p=0.10$} \\
\hline & & $\widehat{\delta}$ & $\widehat{\lambda}$ & $\widehat{\delta}$ & $\widehat{\lambda}$ \\
\hline \multirow[t]{4}{*}{30} & 0.25 & $0.0180(0.0474)$ & $0.1243(0.2448)$ & $-0.0201(0.0501)$ & $-0.1300(0.2488)$ \\
\hline & 0.50 & $0.0287(0.0887)$ & $0.1129(0.2317)$ & $-0.0431(0.1004)$ & $-0.1108(0.2217)$ \\
\hline & 1.50 & $0.2515(0.3429)$ & $0.0538(0.1522)$ & $-0.2842(0.3788)$ & $-0.0530(0.1651)$ \\
\hline & 2.50 & $0.5139(0.6520)$ & $0.0652(0.1484)$ & $-0.5949(0.7340)$ & $-0.0667(0.1540)$ \\
\hline \multirow[t]{4}{*}{150} & 0.25 & $0.0076(0.0281)$ & $0.0198(0.1422)$ & $-0.0054(0.0300)$ & $-0.0295(0.1418)$ \\
\hline & 0.50 & $0.0173(0.0583)$ & $0.0186(0.1326)$ & $-0.0081(0.0632)$ & $-0.0171(0.1299)$ \\
\hline & 1.50 & $0.1292(0.2013)$ & $0.0528(0.1122)$ & $-0.1501(0.2262)$ & $-0.0514(0.1070)$ \\
\hline & 2.50 & $0.3081(0.4130)$ & $0.0603(0.1096)$ & $-0.3475(0.4552)$ & $-0.0600(0.1071)$ \\
\hline \multirow[t]{4}{*}{400} & 0.25 & $0.0056(0.0241)$ & $0.0088(0.1109)$ & $-0.0035(0.0240)$ & $-0.0016(0.1080)$ \\
\hline & 0.50 & $0.0142(0.0485)$ & $0.0008(0.1043)$ & $-0.0075(0.0507)$ & $-0.0064(0.1067)$ \\
\hline & 1.50 & $0.0793(0.1428)$ & $0.0361(0.0958)$ & $-0.0998(0.1652)$ & $-0.0407(0.0974)$ \\
\hline & 2.50 & $0.2050(0.2933)$ & $0.0548(0.0968)$ & $-0.2460(0.3399)$ & $-0.0491(0.0993)$ \\
\hline \multirow[t]{5}{*}{600} & 0.25 & $0.0048(0.0221)$ & $0.0039(0.1050)$ & $-0.0048(0.0222)$ & $-0.0001(0.1039)$ \\
\hline & 0.50 & $0.0120(0.0463)$ & $0.0120(0.0988)$ & $-0.0065(0.0470)$ & $-0.0104(0.0954)$ \\
\hline & 1.50 & $0.0586(0.1225)$ & $0.0259(0.0906)$ & $-0.0745(0.1390)$ & $-0.0302(0.0879)$ \\
\hline & 2.50 & $0.1601(0.2406)$ & $0.0166(0.0888)$ & $-0.2071(0.2941)$ & $-0.0259(0.0906)$ \\
\hline & & \multicolumn{2}{|c|}{$p=0.25$} & \multicolumn{2}{|c|}{$p=0.40$} \\
\hline$n$ & $\delta$ & $\widehat{\delta}$ & $\widehat{\lambda}$ & $\widehat{\delta}$ & $\widehat{\lambda}$ \\
\hline \multirow[t]{4}{*}{30} & 0.25 & $-0.0322(0.0625)$ & $-0.1496(0.2632)$ & $-0.0524(0.0853)$ & $-0.1550(0.2654)$ \\
\hline & 0.50 & $-0.0618(0.1211)$ & $-0.1121(0.2298)$ & $-0.1007(0.1635)$ & $-0.1300(0.2465)$ \\
\hline & 1.50 & $-0.3342(0.4377)$ & $-0.0549(0.1776)$ & $-0.4296(0.5395)$ & $-0.0683(0.1888)$ \\
\hline & 2.50 & $-0.7106(0.8647)$ & $-0.0651(0.1605)$ & $-0.8870(1.0495)$ & $-0.0606(0.1744)$ \\
\hline \multirow[t]{4}{*}{150} & 0.25 & $-0.0081(0.0361)$ & $-0.0385(0.1483)$ & $-0.0147(0.0432)$ & $-0.0405(0.1501)$ \\
\hline & 0.50 & $-0.0163(0.0718)$ & $-0.0139(0.1322)$ & $-0.0316(0.0866)$ & $-0.0293(0.1332)$ \\
\hline & 1.50 & $-0.2016(0.2779)$ & $-0.0554(0.1152)$ & $-0.2488(0.3380)$ & $-0.0498(0.1118)$ \\
\hline & 2.50 & $-0.4300(0.5518)$ & $-0.0612(0.1121)$ & $-0.5518(0.6830)$ & $-0.0598(0.1118)$ \\
\hline \multirow[t]{4}{*}{400} & 0.25 & $-0.0017(0.0273)$ & $-0.0042(0.1139)$ & $-0.0028(0.0315)$ & $-0.0039(0.1131)$ \\
\hline & 0.50 & $-0.0032(0.0563)$ & $-0.0068(0.1068)$ & $-0.0075(0.0661)$ & $-0.0191(0.1068)$ \\
\hline & 1.50 & $-0.1292(0.1974)$ & $-0.0507(0.0986)$ & $-0.1692(0.2431)$ & $-0.0562(0.0994)$ \\
\hline & 2.50 & $-0.3097(0.4059)$ & $-0.0497(0.0984)$ & $-0.3984(0.5129)$ & $-0.0486(0.0998)$ \\
\hline \multirow[t]{4}{*}{600} & 0.25 & $-0.0011(0.0248)$ & $-0.0087(0.1011)$ & $-0.0027(0.0285)$ & $-0.0157(0.1054)$ \\
\hline & 0.50 & $-0.0019(0.0539)$ & $-0.0060(0.0969)$ & $-0.0017(0.0591)$ & $-0.0149(0.0946)$ \\
\hline & 1.50 & $-0.1041(0.1700)$ & $-0.0372(0.0921)$ & $-0.1561(0.2256)$ & $-0.0267(0.0936)$ \\
\hline & 2.50 & $-0.2681(0.3623)$ & $-0.0265(0.0931)$ & $-0.3406(0.4450)$ & $-0.0426(0.0946)$ \\
\hline
\end{tabular}

white blood cell at the time of diagnosis is also recorded. The patients were separated into two groups as: presence or absence of a morphological characteristic of white blood cells. Patients labeled as positive antigen were identified by the pres- 
Table 2 Empirical bias (with MSEs in parentheses) of the ML estimators of $\delta$ and $\lambda=3.0$ from the $R B S$ frailty model under the indicated $n, \delta$ and $p$ for simulated data

\begin{tabular}{|c|c|c|c|c|c|}
\hline \multirow[b]{2}{*}{$n$} & \multirow[b]{2}{*}{$\delta$} & \multicolumn{2}{|c|}{$p=0.00$} & \multicolumn{2}{|c|}{$p=0.10$} \\
\hline & & $\widehat{\delta}$ & $\widehat{\lambda}$ & $\widehat{\delta}$ & $\widehat{\lambda}$ \\
\hline \multirow[t]{4}{*}{30} & 0.25 & $0.0144(0.0400)$ & $-0.6436(0.8083)$ & $-0.0250(0.0553)$ & $-0.7028(0.8855)$ \\
\hline & 0.50 & $0.0324(0.0842)$ & $-0.5471(0.6990)$ & $-0.0402(0.0898)$ & $-0.5946(0.7553)$ \\
\hline & 1.50 & $0.2142(0.3007)$ & $-0.3918(0.5345)$ & $-0.2362(0.3324)$ & $-0.4420(0.5847)$ \\
\hline & 2.50 & $0.4496(0.5773)$ & $-0.3675(0.4964)$ & $-0.5049(0.6455)$ & $-0.3857(0.5163)$ \\
\hline \multirow[t]{4}{*}{150} & 0.25 & $0.0136(0.0273)$ & $-0.3441(0.4645)$ & $-0.0211(0.0455)$ & $-0.4125(0.5411)$ \\
\hline & 0.50 & $0.0297(0.0559)$ & $-0.2925(0.3944)$ & $-0.0367(0.0581)$ & $-0.3071(0.4215)$ \\
\hline & 1.50 & $0.0721(0.1445)$ & $-0.1584(0.2508)$ & $-0.0894(0.1662)$ & $-0.1808(0.2745)$ \\
\hline & 2.50 & $0.2032(0.2972)$ & $-0.1236(0.2101)$ & $-0.2488(0.3513)$ & $-0.1270(0.2165)$ \\
\hline \multirow[t]{4}{*}{400} & 0.25 & $0.0094(0.0242)$ & $-0.2224(0.3140)$ & $-0.0181(0.0351)$ & $-0.2853(0.3826)$ \\
\hline & 0.50 & $0.0241(0.0501)$ & $-0.1918(0.2803)$ & $-0.0363(0.0508)$ & $-0.1959(0.2775)$ \\
\hline & 1.50 & $0.0217(0.0953)$ & $-0.0736(0.1478)$ & $-0.0354(0.1039)$ & $-0.0739(0.1511)$ \\
\hline & 2.50 & $0.1057(0.1860)$ & $-0.0521(0.1242)$ & $-0.1258(0.2165)$ & $-0.0525(0.1278)$ \\
\hline \multirow[t]{5}{*}{600} & 0.25 & $0.0085(0.0232)$ & $-0.1965(0.2832)$ & $-0.0108(0.0298)$ & $-0.2162(0.3138)$ \\
\hline & 0.50 & $0.0214(0.0484)$ & $-0.1630(0.2469)$ & $-0.0306(0.0486)$ & $-0.1637(0.2457)$ \\
\hline & 1.50 & $0.0065(0.0785)$ & $-0.0560(0.1269)$ & $-0.0193(0.0879)$ & $-0.0535(0.1298)$ \\
\hline & 2.50 & $0.0771(0.1512)$ & $-0.0275(0.0995)$ & $-0.0917(0.1714)$ & $-0.0297(0.1048)$ \\
\hline & & \multicolumn{2}{|c|}{$p=0.25$} & \multicolumn{2}{|c|}{$p=0.40$} \\
\hline$n$ & $\delta$ & $\widehat{\delta}$ & $\widehat{\lambda}$ & $\widehat{\delta}$ & $\widehat{\lambda}$ \\
\hline \multirow[t]{4}{*}{30} & 0.25 & $-0.0316(0.0658)$ & $-0.7550(0.9369)$ & $-0.0434(0.0795)$ & $-0.7926(0.9759)$ \\
\hline & 0.50 & $-0.0526(0.1125)$ & $-0.5977(0.7638)$ & $-0.0932(0.1545)$ & $-0.6264(0.7952)$ \\
\hline & 1.50 & $-0.3005(0.3996)$ & $-0.4339(0.5805)$ & $-0.3968(0.5079)$ & $-0.4856(0.6375)$ \\
\hline & 2.50 & $-0.6380(0.7985)$ & $-0.4134(0.5495)$ & $-0.7652(0.9326)$ & $-0.4151(0.5671)$ \\
\hline \multirow[t]{4}{*}{150} & 0.25 & $-0.0298(0.0470)$ & $-0.4070(0.5249)$ & $-0.0302(0.0521)$ & $-0.4231(0.5525)$ \\
\hline & 0.50 & $-0.0405(0.0658)$ & $-0.2979(0.4101)$ & $-0.0580(0.0735)$ & $-0.3080(0.421$ \\
\hline & 1.50 & $-0.1199(0.1999)$ & $-0.1853(0.2831)$ & $-0.1644(0.2469)$ & $-0.1968(0.2991)$ \\
\hline & 2.50 & $-0.3073(0.4209)$ & $-0.1526(0.2423)$ & $-0.4527(0.5931)$ & $-0.1583(0.2573)$ \\
\hline \multirow[t]{4}{*}{400} & 0.25 & $-0.0278(0.0385)$ & $-0.2781(0.3766)$ & $-0.0250(0.0434)$ & $-0.2910(0.3639)$ \\
\hline & 0.50 & $-0.0345(0.0536)$ & $-0.2034(0.2899)$ & $-0.0388(0.0590)$ & $-0.2149(0.277$ \\
\hline & 1.50 & $-0.0638(0.1340)$ & $-0.0908(0.1675)$ & $-0.1081(0.1838)$ & $-0.0985(0.1785)$ \\
\hline & 2.50 & $-0.1807(0.2722)$ & $-0.0554(0.1322)$ & $-0.2709(0.3831)$ & $-0.0782(0.1565)$ \\
\hline \multirow[t]{4}{*}{600} & 0.25 & $-0.0150(0.0335)$ & $-0.2218(0.3157)$ & $-0.0177(0.0373)$ & $-0.2318(0.3091)$ \\
\hline & 0.50 & $-0.0333(0.0493)$ & $-0.1675(0.2425)$ & $-0.0365(0.0540)$ & $-0.1883(0.244$ \\
\hline & 1.50 & $-0.0402(0.1116)$ & $-0.0622(0.1381)$ & $-0.0735(0.1443)$ & $-0.0648(0.1433)$ \\
\hline & 2.50 & $-0.1346(0.2172)$ & $-0.0349(0.1121)$ & $-0.2245(0.3244)$ & $-0.0496(0.1243)$ \\
\hline
\end{tabular}

ence of significant granulation of the leukemic cells in the bone marrow at diagnosis. Factors related to leukemia corresponding to "chemical agents" and "genetic characteristics" were not measured. It motivates the use of a frailty model to cap- 
Table 3 Empirical bias (with MSEs in parentheses) of the ML estimators of $\delta$ and $\lambda=10.0$ from the RBS frailty model under the indicated $n, \delta$ and $p$ for simulated data

\begin{tabular}{|c|c|c|c|c|c|}
\hline \multirow[b]{2}{*}{$n$} & \multirow[b]{2}{*}{$\delta$} & \multicolumn{2}{|c|}{$p=0.00$} & \multicolumn{2}{|c|}{$p=0.10$} \\
\hline & & $\widehat{\delta}$ & $\widehat{\lambda}$ & $\widehat{\delta}$ & $\widehat{\lambda}$ \\
\hline \multirow[t]{4}{*}{30} & 0.25 & $0.0311(0.0541)$ & $-2.5836(3.1092)$ & $-0.0381(0.0542)$ & $-2.7628(2.9962)$ \\
\hline & 0.50 & $0.0366(0.0795)$ & $-1.9971(2.4670)$ & $-0.0533(0.0852)$ & $-2.0266(2.5129)$ \\
\hline & 1.50 & $0.2246(0.3107)$ & $-1.4200(1.8163)$ & $-0.2339(0.3029)$ & $-1.6389(1.8370)$ \\
\hline & 2.50 & $0.4513(0.5748)$ & $-1.3007(1.6595)$ & $-0.4549(0.5804)$ & $-1.5487(1.7312)$ \\
\hline \multirow[t]{4}{*}{150} & 0.25 & $0.0252(0.0474)$ & $-1.6005(1.9594)$ & $-0.0301(0.0479)$ & $-1.5255(1.8792)$ \\
\hline & 0.50 & $0.0320(0.0545)$ & $-1.0724(1.3909)$ & $-0.0428(0.0563)$ & $-1.1615(1.3506)$ \\
\hline & 1.50 & $0.0689(0.1466)$ & $-0.7118(0.9319)$ & $-0.0806(0.1420)$ & $-0.9806(0.9128)$ \\
\hline & 2.50 & $0.1938(0.2991)$ & $-0.5731(0.7910)$ & $-0.2003(0.2969)$ & $-0.6778(0.7844)$ \\
\hline \multirow[t]{4}{*}{400} & 0.25 & $0.0212(0.0365)$ & $-1.0571(1.3193)$ & $-0.0232(0.0359)$ & $-1.0114(1.2847)$ \\
\hline & 0.50 & $0.0214(0.0511)$ & $-0.7708(0.9990)$ & $-0.0313(0.0511)$ & $-0.8650(0.9840)$ \\
\hline & 1.50 & $0.0157(0.0864)$ & $-0.4063(0.5767)$ & $-0.0323(0.0826)$ & $-0.5121(0.5807)$ \\
\hline & 2.50 & $0.0895(0.1668)$ & $-0.3082(0.4477)$ & $-0.0916(0.1620)$ & $-0.4006(0.4514)$ \\
\hline \multirow[t]{5}{*}{600} & 0.25 & $0.0173(0.0311)$ & $-0.8929(1.1393)$ & $-0.0207(0.0306)$ & $-0.9836(1.1267)$ \\
\hline & 0.50 & $0.0134(0.0508)$ & $-0.6830(0.8789)$ & $-0.0255(0.0498)$ & $-0.7804(0.8696)$ \\
\hline & 1.50 & $0.0141(0.0735)$ & $-0.3244(0.4692)$ & $-0.0231(0.0733)$ & $-0.3281(0.4621)$ \\
\hline & 2.50 & $0.0497(0.1279)$ & $-0.2517(0.3732)$ & $-0.0625(0.1386)$ & $-0.3437(0.3648)$ \\
\hline & & \multicolumn{2}{|c|}{$p=0.25$} & \multicolumn{2}{|c|}{$p=0.40$} \\
\hline$n$ & $\delta$ & $\widehat{\delta}$ & $\widehat{\lambda}$ & $\widehat{\delta}$ & $\widehat{\lambda}$ \\
\hline \multirow[t]{4}{*}{30} & 0.25 & $-0.0688(0.0577)$ & $-2.8324(3.0606)$ & $-0.0905(0.0708)$ & $-2.9354(3.2976)$ \\
\hline & 0.50 & $-0.0744(0.0847)$ & $-2.1751(2.6420)$ & $-0.0802(0.1124)$ & $-2.3151(2.6025)$ \\
\hline & 1.50 & $-0.2528(0.3061)$ & $-1.9400(1.9445)$ & $-0.2861(0.3304)$ & $-1.9660(1.9507)$ \\
\hline & 2.50 & $-0.4894(0.6311)$ & $-1.7433(1.7000)$ & $-0.4905(0.6098)$ & $-1.8130(1.7998)$ \\
\hline \multirow[t]{4}{*}{150} & 0.25 & $-0.0456(0.0453)$ & $-1.6193(1.8963)$ & $-0.0698(0.0491)$ & $-1.8798(1.8341)$ \\
\hline & 0.50 & $-0.0484(0.0567)$ & $-1.4136(1.4209)$ & $-0.0712(0.0618)$ & $-1.5296(1.4425)$ \\
\hline & 1.50 & $-0.0935(0.1443)$ & $-0.9955(0.9381)$ & $-0.0995(0.1581)$ & $-1.0138(0.9350)$ \\
\hline & 2.50 & $-0.2027(0.3017)$ & $-0.7259(0.8469)$ & $-0.2153(0.3152)$ & $-0.8857(0.8054)$ \\
\hline \multirow[t]{4}{*}{400} & 0.25 & $-0.0278(0.0368)$ & $-1.4530(1.3170)$ & $-0.0434(0.0420)$ & $-1.6708(1.3467)$ \\
\hline & 0.50 & $-0.0323(0.0519)$ & $-0.9535(0.9751)$ & $-0.0571(0.0525)$ & $-0.9554(0.9789)$ \\
\hline & 1.50 & $-0.0558(0.0890)$ & $-0.4221(0.5815)$ & $-0.0845(0.0946)$ & $-0.4444(0.6167)$ \\
\hline & 2.50 & $-0.1010(0.1850)$ & $-0.4133(0.5094)$ & $-0.1991(0.1866)$ & $-0.4502(0.4973)$ \\
\hline \multirow[t]{4}{*}{600} & 0.25 & $-0.0246(0.0322)$ & $-0.9938(1.1427)$ & $-0.0303(0.0356)$ & $-0.9997(1.1321)$ \\
\hline & 0.50 & $-0.0354(0.0509)$ & $-0.7971(0.8707)$ & $-0.0392(0.0506)$ & $-0.8887(0.8850)$ \\
\hline & 1.50 & $-0.0398(0.0751)$ & $-0.3763(0.4795)$ & $-0.0443(0.0818)$ & $-0.4229(0.4622)$ \\
\hline & 2.50 & $-0.0748(0.1001)$ & $-0.3657(0.2823)$ & $-0.0822(0.1391)$ & $-0.3949(0.3695)$ \\
\hline
\end{tabular}

ture the effect of such factors. Table 4 reports the ML estimates of the RBS-EXP, RBS-WE, GA-EX and GA-WE frailty model parameters (with estimated asymptotic standard errors in parentheses), that is, models with frailties RBS and GA 
Table 4 ML estimate with standard errors in parentheses and selection criteria for the indicated model fitted to leukemia data

\begin{tabular}{lcccccc}
\hline Frailty model & Baseline & Parameter & ML estimate & AIC & BIC & BF \\
\hline RBS & EXP & $\delta$ & $1.5640(1.1497)$ & & & \\
& & $\lambda$ & $0.0619(0.0304)$ & 290.0783 & 292.9463 & 9.2453 \\
& WE & $\delta$ & $0.0226(0.0179)$ & & & \\
& & $\alpha$ & $4.5541(1.0941)$ & & & \\
GA & $\beta$ & $2.7008(0.4203)$ & 280.8330 & 283.7010 & - \\
& EXP & $\gamma$ & $0.5019(0.3922)$ & & & \\
& & $\lambda$ & $0.0432(0.0182)$ & 293.8415 & 296.7095 & 13.009 \\
& WE & $\gamma$ & $0.2176(0.6974)$ & & & \\
& & $\alpha$ & $24.3606(14.508)$ & & & \\
& & $\beta$ & $0.7999(0.2553)$ & 290.9419 & 295.7060 & 12.005 \\
\hline
\end{tabular}

Table 5 Estimated frailty variance for the indicated model with leukemia data

\begin{tabular}{|c|c|c|c|c|}
\hline \multirow[b]{2}{*}{ Variance } & \multicolumn{4}{|c|}{ Model } \\
\hline & RBS-EXP & RBS-WE & GA-EXP & GA-WE \\
\hline$\widehat{\operatorname{Var}}[U]$ & 1.2363 & 4.8247 & 0.5019 & 0.2176 \\
\hline
\end{tabular}

distributed with EXP and WE baselines. In this table, values for Akaike (AIC) and Bayesian (BIC) information criteria, and Bayes factor (BF) are provided. We use the $\mathrm{BF}$ to evaluate the magnitude of the difference between two BIC values; see Kass and Raftery (1995). We compute the AIC and BIC in all models, but the BF is obtained for comparing the RBS and GA models with the same baseline HR, that is, RBS-EXP model versus GA-EXP model, and RBS-WE model versus GA-WE model. Decision about the best fit is made according to the interpretation of the BF presented in Table 6 of Leiva et al. (2015b). Table 4 indicates that the RBS frailty model with WE baseline provides the best overall fit in terms of AIC, BIC and BF. The estimated variance of the RBS and GA frailty models are respectively given by

$$
\widehat{\operatorname{Var}}[U]=(2 \widehat{\delta}+5) /(\widehat{\delta}+1)^{2}, \quad \widehat{\operatorname{Var}}[U]=\widehat{\gamma} .
$$

Based on Table 4 and variances given in Equation (3.1), Table 5 summarizes the corresponding estimated variances. From this table, note that the estimated frailty variances are different from zero. This indicates the presence of unobserved heterogeneity. Notice that the estimated frailty variance is greater in the RBS model than in the GA model, in all cases, indicating that the RBS model is better in terms of capturing the heterogeneity unobserved in the data. In addition, from Table 4, note that the RBS-WE model provides the best fit compared to the RBS-EXP, GAEXP and GA-WE models based on the values of AIC, BIC and BF. 


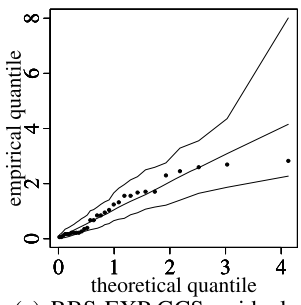

(a) RBS-EXP GCS residual

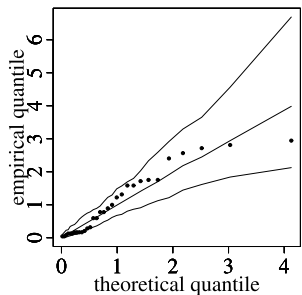

(e) GA-EXP GCS residual

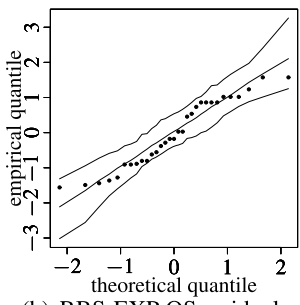

(b) RBS-EXP QS residual

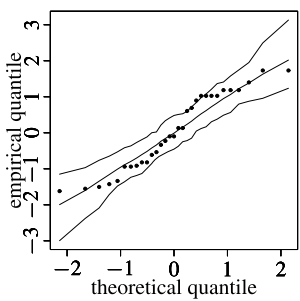

(f) GA-EXP QS residual

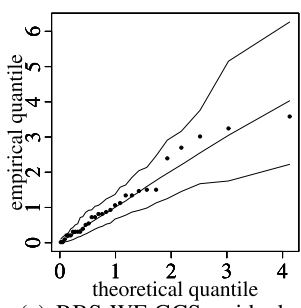

(c) RBS-WE GCS residual

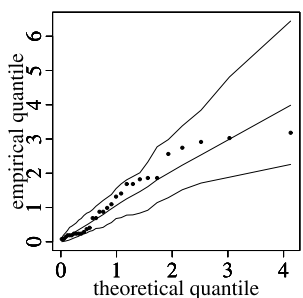

(g) GA-WE GCS residual

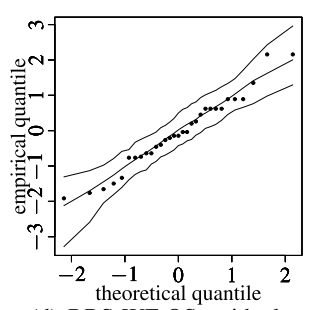

(d) RBS-WE QS residual

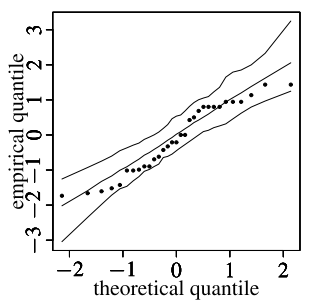

(h) GA-WE QS residual

Figure $2 Q Q$ plot with simulated envelope under the indicated residual and model for leukemia data.
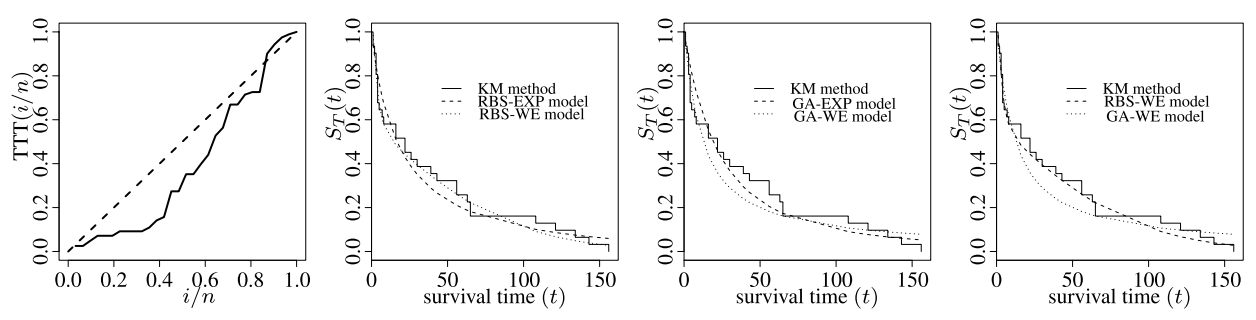

Figure 3 TTT plot (left) and fitted SFs by the indicated model (right) for leukemia data.

Figure 2 shows the QQ plots with simulated envelope for the GCS and QS residuals. These plots allow us to check graphically whether the GCS and QS residuals follow the $\operatorname{EXP}(1)$ and $\mathrm{N}(0,1)$ distributions or not, respectively. From Figure 2, note that these residuals present a good agreement with their corresponding target distributions.

In Figure 3, we display the total time on test (TTT) curve and estimated (fitted) SFs based on the Kaplan-Meier (KM) estimator and the RBS-EXP, RBS-WE, GAEXP and GA-WE frailty models. The TTT plot allows us to characterize the shape of an HR (constant, increasing, decreasing, bathtub-shaped, or inverse bathtubshaped), whereas the plot of the SFs permits us to compare the empirical and fitted SFs of the data; see, for example, Figure 1 in Azevedo et al. (2012) for different theoretical shapes of scaled TTT curves. Figure 3(left) suggests a decreasing HR. Therefore, a natural choice for the baseline seems to be the WE distribution since it is used to model monotone HR, that is, constant, increasing and decreasing HR. 
Table 6 ML estimates with standard errors in parentheses and selection criteria for the indicated model fitted to lung data

\begin{tabular}{lcccccc}
\hline Frailty model & Baseline & Parameter & ML estimate & AIC & BIC & BF \\
\hline RBS & EXP & $\delta$ & $0.9539(0.1906)$ & & & \\
& & $\lambda$ & $0.0202(0.0037)$ & 1514.3670 & 1520.8070 & 12.418 \\
& WE & $\delta$ & $1.6255(0.5449)$ & & & \\
& & $\alpha$ & $68.3897(12.4730)$ & & & \\
GA & EX & $\gamma$ & $1.1920(0.10631)$ & 1499.6290 & 1508.3890 & - \\
& & $\lambda$ & $1.6837(6.0740)$ & & & \\
& WE & $\gamma$ & $1.6360(0.6701)$ & & & \\
& & $\alpha$ & $44.1654(9.4356)$ & & & \\
& & $\beta$ & $1.4445(0.24325)$ & 1517.2260 & 1525.9860 & 17.597 \\
\hline
\end{tabular}

Table 7 Estimated frailty variance for the indicated model with lung data

\begin{tabular}{|c|c|c|c|c|}
\hline \multirow[b]{2}{*}{ Variance } & \multicolumn{4}{|c|}{ Model } \\
\hline & RBS-EXP & RBS-WE & GA-EXP & GA-WE \\
\hline$\widehat{\operatorname{Var}}[U]$ & 1.8094 & 1.1969 & 1.6837 & 1.6360 \\
\hline
\end{tabular}

The results indicate that, as expected, the best fit to the data is provided by the RBS-WE model. It is important to highlight that the overall results suggest that the RBS frailty model is better than the GA frailty model for all baselines considered, indicating the potentiality of the new model in describing frailty data.

\section{Second case study: Lung cancer data}

This data set is related to the survival times on 137 advanced lung cancer patients, whose set we name "lung data"; see Kalbfleisch and Prentice (2002). The censoring proportion is $p=0.0657$ (6.57\%). The ML estimates of the model parameters (with standard errors in parentheses), AICs, BICs and BFs are summarized in Table 6. Comparing the information criteria, we notice that the RBS frailty model with WE baseline has the smallest AIC and BIC values, suggesting that it provides a good fit for these data. Also, the BF supports this result. The summary of the estimated frailty variances is given in Table 7. From this table, note that the estimated variances of the RBS and GA frailty models indicate the presence of unobserved heterogeneity for both baselines. Moreover, the results from Table 6 show that the RBS frailty model presents a better fit than the GA frailty model.

The QQ plots with simulated envelope for the GCS and QS residuals are displayed in Figure 4. These graphical plots show the notorious superiority, in terms 


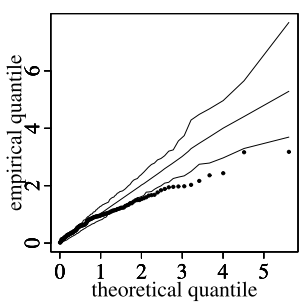

(a) RBS-EXP GCS residual

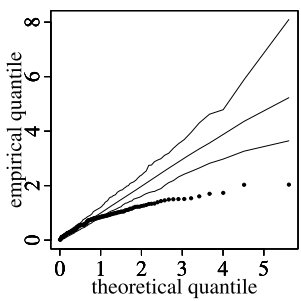

(e) GA-EXP GCS residual

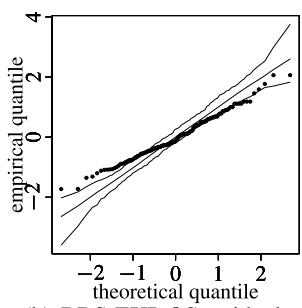

(b) RBS-EXP QS residual

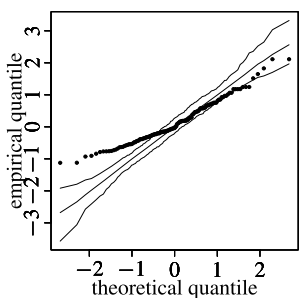

(f) GA-EXP QS residual

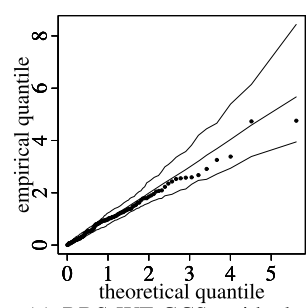

(c) RBS-WE GCS residual

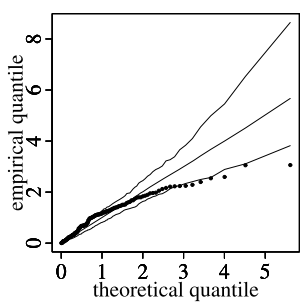

(g) GA-WE GCS residual

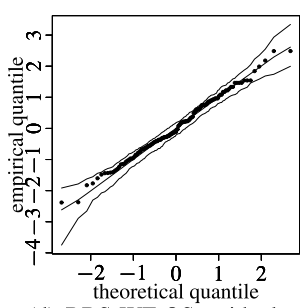

(d) RBS-WE QS residual

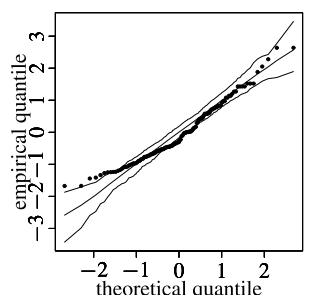

(h) GA-WE QS residual

Figure $4 Q Q$ plot with simulated envelope under the indicated residual and model for lung data.
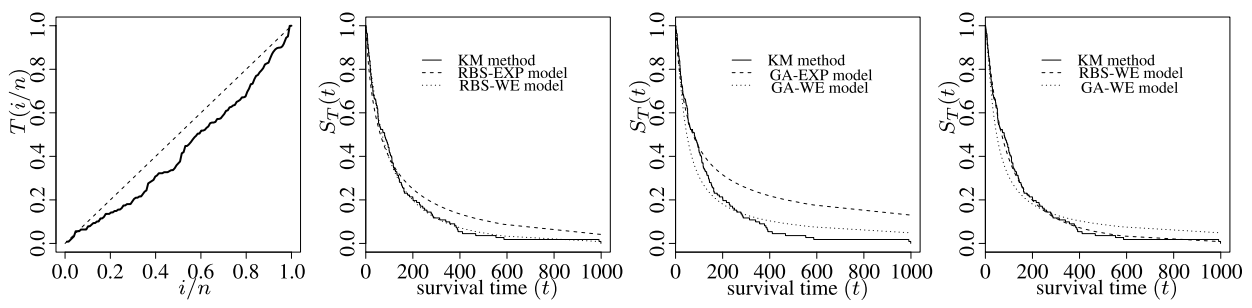

Figure 5 TTT plot (left) and fitted SFs by the indicated model (right) for lung data.

of fitting to the data, of the RBS frailty model with WE baseline over all other models.

Figure 5 shows the TTT plot and fitted SF by the KM method. Note that Figure 5(left) suggests a decreasing HR for lung data. The fitted SFs presented in Figure 5(right) confirms graphically the superiority of the RBS frailty model with WE baseline suggested by the results in Table 6 .

\section{Concluding remarks}

Non-measurable biological variations among patients can be presented, which introduces heterogeneity among them. For instance, some patients may have a genetic disposition with respect to certain disease, having an increasing risk of developing it compared to others. The heterogeneity therefore affects the observed survival times. The inclusion of a frailty parameter in the survival data modeling brings additional information that may be useful in practice. Thus, a source 
of unobserved heterogeneity that is not captured by explanatory variables can be introduced by a frailty component in the hazard rate structure. Then, the effects of omitted explanatory variables may be now captured. We have proposed and developed a new frailty model based on a Birnbaum-Saunders distribution. In the model, this distribution is employed to describe the unobserved frailty. Due to its properties and features, the new model can be a good alternative to the gamma frailty model. Using the Laplace transform, we have derived explicitly the unconditional hazard rate and survival function. A Monte Carlo simulation study has shown that the estimates based on the maximum likelihood method of the model parameters tend to their true values, whereas the distributions of these estimators converges to normality, when the sample size increases, as expected. We have considered two types of residuals to assess the goodness of fit of the model to the data. Also, we have obtained the observed information matrix analytically, which facilitates the direct computation of the corresponding estimated asymptotic standard errors. We have applied the proposed methodology to uncensored and censored real-world data sets. These two data sets are related to survival times of patients who died from acute myelogenous leukemia and advanced lung cancer, respectively. The applications have shown the potential of the new model. The precision parameter of the Birnbaum-Saunders distribution, which measures the effect of the time in the corresponding frailty model, has been significant and positive. Then, we have an increasing hazard rate that could help medical doctors to predict the occurrence to the event of interest anticipatively, which does not happen in the model with no frailty. The $\mathrm{R}$ codes used in this paper, as well as the data sets, are available under request from the authors. An $\mathrm{R}$ package is currently under progress and we hope to report it in a future paper. The proposed methodology can be applied to other distributions. However, mathematical difficulties may be found for other distributions when doing inference over the model parameters. In addition, influence diagnostic tools may also be derived for this type of models in order to evaluate the effect of atypical observations on it, as well as the inclusion of multivariate aspects in frailty models and/or spatio-temporal structures; see Marchant, Leiva and Cysneiros (2016a), Marchant et al. (2016b) and Garcia-Papani et al. (2017). Thus, the present study leaves some open topics to be addressed in the future.

\section{Acknowledgments}

The authors thank the Editors and reviewers for their constructive comments on an earlier version of this manuscript. This research work was partially supported by CNPq, CAPES and FAPESP grants from Brazil and by FONDECYT 1160868 grant from the Chilean government. 


\section{References}

Aalen, O., Borgan, O. and Gjessing, H. (2008). Survival and Event History Analysis: A Process Point of View. New York: Springer. MR2449233

Aalen, O. and Tretli, S. (1999). Analysing incidence of testis cancer by means of a frailty model. Cancer Causes and Control 10, 285-292.

Azevedo, C., Leiva, V., Athayde, E. and Balakrishnan, N. (2012). Shape and change point analyses of the Birnbaum-Saunders-t hazard rate and associated estimation. Computational Statistics and Data Analysis 56, 3887-3897. MR2957839

Baker, P. and Henderson, R. (2005). Small sample bias in the gamma frailty model for univariate survival. Lifetime Data Analysis 11, 265-284. MR2158785

Barros, M., Paula, G. and Leiva, V. (2008). A new class of survival regression models with heavytailed errors: Robustness and diagnostics. Lifetime Data Analysis 14, 316-332. MR2516848

Birnbaum, Z. W. and Saunders, S. C. (1969). A new family of life distributions. Journal of Applied Probability 6, 319-327. MR0253493

Brent, R. (1973). Algorithms for Minimization Without Derivatives. New Jersey: Prentice-Hall. MR0339493

Clayton, D. (1991). A Monte Carlo method for Bayesian inference in frailty models. Biometrics 47, 467-485.

Cox, D. R. (1972). Regression models and life-tables. Journal of the Royal Statistical Society B 34, 187-220. MR0341758

Cox, D. R. and Hinkley, D. V. (1974). Theoretical Statistics. London: Chapman and Hall. MR0370837

Cox, D. R. and Snell, E. (1968). A general definition of residuals. Journal of the Royal Statistical Society B 2, 248-275. MR0237052

Desmond, A. (1985). Stochastic models of failure in random environments. Canadian Journal of Statistics 13, 171-183. MR0818323

Dunn, P. and Smyth, G. (1996). Randomized quantile residuals. Journal of Computational and Graphical Statistics 5, 236-244.

Efron, B. and Hinkley, D. V. (1978). Assessing the accuracy of the maximum likelihood estimator: Observed vs. expected Fisher information. Biometrika 65, 457-487. MR0521817

Elbers, C. and Ridder, G. (1982). True and spurious duration dependence: The identificability of the proportional hazard model. Review of Economic Studies 49, 403-409. MR0667501

Feigl, P. and Zelen, M. (1965). Estimation of exponential survival probabilities with concomitant information. Biometrics 21, 826-837.

Garcia-Papani, F., Uribe-Opazo, M. A., Leiva, V. and Aykroyd, R. G. (2017). Birnbaum-Saunders spatial modelling and diagnostics applied to agricultural engineering data. Stochastic Environmental Research and Risk Assessment 31, 105-124.

Henderson, R. and Oman, P. (1999). Effect of frailty on marginal regression estimates in survival analysis. Journal of the Royal Statistical Society B 61, 367-379. MR1680322

Hougaard, P. (1984). Life table methods for heterogeneous populations: Distributions describing the heterogeneity. Biometrika 71, 75-84. MR0738328

Hougaard, P. (1995). Frailty models for survival data. Lifetime Data Analysis 1, 255-273.

Kalbfleisch, J. and Prentice, R. (2002). The Statistical Analysis of Failure Time Data. New York: Wiley. MR1924807

Kass, R. and Raftery, A. (1995). Bayes factors. Journal of the American Statistical Association 90, 773-795. MR3363402

Klein, J. and Moeschberger, M. (2003). Survival Analysis: Techniques for Censored and Truncated Data. New York: Springer. 
Kotz, S., Leiva, V. and Sanhueza, A. (2010). Two new mixture models related to the inverse Gaussian distribution. Methodology and Computing in Applied Probability 12, 199-212. MR2580102

Lange, K. (2001). Numerical Analysis for Statisticians. New York: Springer. MR1681963

Lawless, J. (2003). Statistical Models and Methods for Lifetime Data. New York: Wiley. MR1940115

Leao, J., Leiva, V., Saulo, H. and Tomazella, V. (2017). Birnbaum-Saunders frailty regression models: Diagnostics and application to medical data. Biometrical Journal 59, 291-314.

Leiva, V. (2016). The Birnbaum-Saunders Distribution. New York: Academic Press. Available at https://mathscinet.ams.org/mathscinet-getitem?mr=3430824. MR3430824

Leiva, V., Ferreira, M., Gomes, M. I. and Lillo, C. (2016a). Extreme value Birnbaum-Saunders regression models applied to environmental data. Stochastic Environmental Research and Risk Assessment 30, 1045-1058.

Leiva, V., Marchant, C., Ruggeri, F. and Saulo, H. (2015a). A criterion for environmental assessment using Birnbaum-Saunders attribute control charts. Environmetrics 26, 463-476. MR3415566

Leiva, V., Ruggeri, F., Saulo, H. and Vivanco, J. F. (2017). A methodology based on the BirnbaumSaunders distribution for reliability analysis applied to nano-materials. Reliability Engineering and System Safety 157, 192-201.

Leiva, V., Santos-Neto, M., Cysneiros, F. J. A. and Barros, M. (2014a). Birnbaum-Saunders statistical modelling: A new approach. Statistical Modelling 14, 21-48. MR3179546

Leiva, V., Santos-Neto, M., Cysneiros, F. J. A. and Barros, M. (2016b). A methodology for stochastic inventory models based on a zero-adjusted Birnbaum-Saunders distribution. Applied Stochastic Models in Business and Industry 32, 74-89. MR3460890

Leiva, V., Saulo, H., Leão, J. and Marchant, C. (2014b). A family of autoregressive conditional duration models applied to financial data. Computational Statistics and Data Analysis 79, 175191. MR3227995

Leiva, V. and Saunders, S. C. (2015). Cumulative damage models. Wiley StatsRef: Statistics Reference Online.

Leiva, V., Tejo, M., Guiraud, P., Schmachtenberg, O., Orio, P. and Marmolejo, F. (2015b). Modeling neural activity with cumulative damage distributions. Biological Cybernetics 109, 421-433. MR3397465

Lemonte, A. (2013). A new extension of the Birnbaum Saunders distribution. Brazilian Journal of Probability and Statistics 27, 133-149. MR3028800

Lillo, C., Leiva, V., Nicolis, O. and Aykroyd, R. G. (2018). L-moments of the Birnbaum-Saunders distribution and its extreme value version: Estimation, goodness of fit and application to earthquake data. Journal of Applied Statistics 45, 187-209.

Marchant, C., Leiva, V. and Cysneiros, F. J. A. (2016a). A multivariate log-linear model for Birnbaum-Saunders distributions. IEEE Transactions on Reliability 65, 816-827.

Marchant, C., Leiva, V., Cysneiros, F. J. A. and Vivanco, J. F. (2016b). Diagnostics in multivariate generalized Birnbaum-Saunders regression models. Journal of Applied Statistics 43, 2829-2849. MR3546117

Nocedal, J. and Wright, S. (1999). Numerical Optimization. New York: Springer. MR1713114

R-Team (2016). R: A Language and Environment for Statistical Computing. Vienna: R Foundation for Statistical Computing.

Rieck, J. and Nedelman, J. (1991). A log-linear model for the Birnbaum-Saunders distribution. Technometrics 3, 51-60.

Sánchez, L., Leiva, V., Caro-Lopera, F. and Cysneiros, F. (2015). On matrix-variate BirnbaumSaunders distributions and their estimation and application. Brazilian Journal of Probability and Statistics 29, 790-812. MR3397394

Santos-Neto, M., Cysneiros, F. J. A., Leiva, V. and Ahmed, S. (2012). On new parameterizations of the Birnbaum-Saunders distribution. Pakistan Journal of Statistics 28, 1-26. MR2931825 
Santos-Neto, M., Cysneiros, F. J. A., Leiva, V. and Barros, M. (2014). On new parameterizations of the Birnbaum-Saunders distribution and its moments, estimation and application. REVSTAT Statistical Journal 12, 247-272. MR3301849

Santos-Neto, M., Cysneiros, F. J. A., Leiva, V. and Barros, M. (2016). Reparameterized BirnbaumSaunders regression models with varying precision. Electronic Journal of Statistics 10, 28252855. MR3553913

Saulo, H., Leão, J., Leiva, V. and Aykroyd, R. G. (2019). Birnbaum-Saunders autoregressive conditional duration models applied to high-frequency financial data. Statistical Papers. To appear. Available at https://doi.org/10.1007/s00362-017-0888-6.

Saulo, H., Leiva, V., Ziegelmann, F. A. and Marchant, C. (2013). A nonparametric method for estimating asymmetric densities based on skewed Birnbaum-Saunders distributions applied to environmental data. Stochastic Environmental Research and Risk Assessment 27, 1479-1491.

Sinha, D. and Dey, D. (1997). Semiparametric Bayesian analysis of survival data. Journal of the American Statistical Association 92, 1195-1212. MR1482151

Smith, J. Q. (1985). Diagnostic checks of non-standard time series models. Journal of Forecasting 4 , 283-291.

Stasinopoulos, D. and Rigby, R. (2007). Generalized additive models for location, scale and shape (GAMLSS). Journal of Statistical Software 23, 1-46. MR2137253

Vanegas, L. H. and Paula, G. A. (2016a). An extension of log-symmetric regression models: R codes and applications. Journal of Statistical Simulation and Computation 86, 1709-1735. MR3473841

Vanegas, L. H. and Paula, G. A. (2016b). Log-symmetric distributions: Statistical properties and parameter estimation. Brazilian Journal of Probability and Statistics 30, 196-220. MR3481101

Vaupel, J., Manton, K. and Stallard, E. (1979). The impact of heterogeneity in individual frailty on the dynamics of mortality. Demography 16, 439-454.

Wanke, P. and Leiva, V. (2015). Exploring the potential use of the Birnbaum-Saunders distribution in inventory management. Mathematical Problems in Engineering 2015, 827246. MR3421264

Wienke, A. (2011). Frailty Models in Survival Analysis. London: Chapman and Hall. MR2682965

Jeremias Leão

Department of Statistics

Universidade Federal do Amazonas

Av. Rodrigo Otávio, n. 6.200

Campus Universitário Sen. Arthur V. Filho

Setor Norte, Coroado I

Manaus, AM 69077-000

Brazil

E-mail: leaojeremiass@gmail.com

Helton Saulo

Department of Statistics

Universidade de Brasília

CIC/EST, Campus Darcy Ribeiro

Brasília, DF 70910-900

Brazil

E-mail: heltonsaulo@gmail.com
Víctor Leiva

School of Industrial Engineering

Pontificia Universidad Católica de Valparaíso

Av. Brasil 2241

Valparaíso 2362807

Chile

E-mail: victorleivasanchez@gmail.com URL: www.victorleiva.cl

Vera Tomazella

Department of Statistics

Universidade Federal de São Carlos

Rodovia Washington Luis, km 235

São Carlos, SP 13565-905

Brazil

E-mail: veratomazella@gmail.com 